\title{
Outcomes of COVID-19 in a cohort of pediatric patients with rheumatic diseases
}

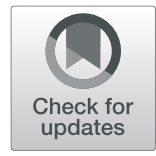

\author{
D. Sofia Villacis-Nunez ${ }^{1,2^{*}}$ (D, Christina A. Rostad ${ }^{3,2}$, Kelly Rouster-Stevens ${ }^{1,2}$, Arezou Khosroshahi ${ }^{4}$, \\ Shanmuganathan Chandrakasan ${ }^{5,2}$ and Sampath Prahalad $d^{1,2,6}$
}

\begin{abstract}
Background: There are few reports of COVID-19 in pediatric patients with rheumatic diseases. This study describes the clinical presentation and outcomes of COVID-19 in this population.

Methods: We analyzed a single-center case series of pediatric patients with rheumatic diseases and laboratoryconfirmed COVID-19. Demographic, baseline and COVID-19 associated clinical features were compared between ambulatory and hospitalized patients using univariate analysis.

Results: Fifty-five cases were identified: $45(81.8 \%)$ in the ambulatory group and 10 (18.2\%) hospitalized. African American race (OR 7.78; 95\% Cl [1.46-55.38]; $p=0.006)$ and cardiovascular disease (OR 19.40; 95\% Cl 2.45-254.14; $p=0.001)$ predominated in hospitalized patients. Active rheumatic disease (OR 11.83; 95\% Cl 1.43-558.37; $p=0.01)$, medium/high-dose corticosteroid use (OR 14.12; 95\% Cl [2.31-106.04]; $p=0.001$ ), mycophenolate use (OR 8.84; 95\% $\mathrm{Cl}$ [1.64-63.88]; $p=0.004)$, rituximab use (OR 19.40; 95\% Cl [2.45-254.14]; $p=0.001)$ and severe immunosuppression (OR 34.80; 95\% Cl [3.94-1704.26]; $\mathrm{p}=<0.001$ ) were associated with increased odds of hospitalization. Fever (OR 7.78; 95\% Cl [1.46-55.38]; $p=0.006)$, dyspnea (OR 26.28; 95\% Cl [2.17-1459.25]; $p=0.003)$, chest pain (OR 13.20; $95 \% \mathrm{Cl}$ [1.53-175.79]; $p=0.007)$, and rash (OR 26.28; $95 \% \mathrm{Cl}[2.17-1459.25] ; \mathrm{p}=0.003)$ were more commonly observed in hospitalized patients. Rheumatic disease flares were almost exclusive to hospitalized patients (OR 55.95; 95\% Cl [5.16-3023.74]; $p<0.001) .$. One patient did not survive.

Conclusions: Medium/high-dose corticosteroid, mycophenolate and rituximab use, and severe immunosuppression were risk factors for hospitalization. Fever, dyspnea, chest pain, and rash were high-risk symptoms for hospitalization. Rheumatic disease activity and flare could contribute to the need for hospitalization.
\end{abstract}

Keywords: Rheumatic diseases, COVID-19, Pediatric rheumatology

\section{Background}

COVID-19 results from infection with SARS-CoV-2, a novel coronavirus, and has been reported to have a milder disease course in children compared to adults $[1,2]$. However, the known susceptibility to infections in patients with rheumatic diseases has been of great concern for pediatric rheumatologists during the

\footnotetext{
* Correspondence: dvillac@emory.edu

${ }^{1}$ Department of Pediatrics, Division of Pediatric Rheumatology, Emory

University School of Medicine, Atlanta, USA

${ }^{2}$ Children's Healthcare of Atlanta, Atlanta, USA

Full list of author information is available at the end of the article
}

pandemic [3, 4]. Outcomes from the largest case series of adults with rheumatic diseases reported $91 \%$ survival, despite a $50 \%$ hospitalization rate [5]. Although some studies of COVID-19 in rheumatic disease patients include a few pediatric cases, with overall favorable outcomes, pediatric data remain scarce [6-9]. The aim of our study was to analyze the clinical presentation and outcomes of COVID-19 in a case series of pediatric patients with rheumatic diseases, and to identify risk factors associated with adverse outcomes.

\section{BMC}

(c) The Author(s). 2021 Open Access This article is licensed under a Creative Commons Attribution 4.0 International License, which permits use, sharing, adaptation, distribution and reproduction in any medium or format, as long as you give appropriate credit to the original author(s) and the source, provide a link to the Creative Commons licence, and indicate if changes were made. The images or other third party material in this article are included in the article's Creative Commons licence, unless indicated otherwise in a credit line to the material. If material is not included in the article's Creative Commons licence and your intended use is not permitted by statutory regulation or exceeds the permitted use, you will need to obtain permission directly from the copyright holder. To view a copy of this licence, visit http://creativecommons.org/licenses/by/4.0/ The Creative Commons Public Domain Dedication waiver (http://creativecommons.org/publicdomain/zero/1.0/) applies to the data made available in this article, unless otherwise stated in a credit line to the data. 


\section{Methods}

Pediatric patients (0-21 years) with rheumatic diseases followed at Children's Healthcare of Atlanta (CHOA) who had laboratory-confirmed SARS-CoV-2 infection from May 2020 to January 2021 were retrospectively identified. Diagnosis was established either through patient/family report of positive SARS-CoV-2 test (serology or polymerase chain reaction (PCR)) or by positive test performed at CHOA. The present study was approved by the Institutional Review Board (IRB) at CHOA via waiver of informed consent (IRB number STUDY00000771).

Cases were classified into two groups based on need for hospitalization. Demographic (age, sex, race/ethnicity), and clinical data (baseline rheumatic disease diagnosis/activity, comorbidities, medications, degree of immunosuppression, COVID-19 exposure/symptoms, immunosuppression management, and outcomes) were collected through chart review.

Rheumatic disease activity was assessed using the Systemic Lupus Erythematosus Disease Activity Index-2000 (SLEDAI-2 K) and Juvenile Arthritis Disease Activity Score (JADAS) when indicated. If unable to calculate, physician assessment of disease activity was utilized. Active disease was defined as a SLEDAI-2 K score $\geq 6$, JADAS-10 score $>2$, or rheumatologist assessment indicating active disease.

The level of immunosuppression was defined as severe if the subject reported use of one or more of: intravenous (IV) cyclophosphamide (within 3 months), rituximab (within 6 months), medium/high-dose ( $\geq 10 \mathrm{mg} /$ day) oral corticosteroids (CS), outpatient IV CS. Patients on immunomodulators not meeting these criteria were considered mild to moderately immunosuppressed.

Demographic and clinical features were compared between groups using Fisher exact test (with corresponding Odds ratios (OR) and 95\% confidence intervals (CI)) for categorical variables, and Mann-U-Whitney test for continuous variables, employing $\mathrm{R}$ software. $P$ values $\leq 0.05$ were considered statistically significant.

\section{Results}

\section{Patient cohort, demographics, and baseline clinical features}

Fifty-five cases were identified: 10 (18.2\%) required hospitalization and $45(81.8 \%)$ received ambulatory care. Demographics and baseline clinical features are outlined in Table 1. Age was similar between groups. The racial and ethnic distribution differed between groups: a higher proportion of African American patients were encountered in the hospitalized group (OR 7.78, 95\% CI [1.46-55.38]; $p=0.006$ ), while Caucasian patients predominated in the ambulatory group (OR 0.14, 95\% CI [0.01-0.83]; $p=0.01$ ).
The frequency of underlying comorbidities was comparable between groups. Reported comorbidities consisted of: asthma $(n=10)$, hypertension $(n=7)$, obesity $(n=5)$, inflammatory bowel disease $(n=4)$, anemia of chronic disease $(n=2)$, immunoglobulin G deficiency $(n=2)$, hypothyroidism $(n=2)$, end-stage renal disease on chronic dialysis $(n=2)$, and one patient each had pulmonary hypertension, chronic kidney disease, post-renal transplant status, left ventricular hypertrophy, chronic diarrhea, cholestasis, adrenal insufficiency, hyperparathyroidism, thyroid disease, chronic neutropenia, sickle cell disease, recurrent infections, chronic deep venous thrombosis, and Wilms tumor. No smoking or diabetes were identified in either group. Patients with cardiovascular disease, namely left ventricular hypertrophy and hypertension, were more likely to be in the hospitalized group (OR 19.40, 95\% CI [2.45-254.14]; $p=0.001)$. No significant differences were observed regarding the presence of the remaining specific comorbidities between groups.

Juvenile idiopathic arthritis (JIA), systemic lupus erythematosus (SLE), and juvenile dermatomyositis (JDM) were the most common primary rheumatic diagnoses among children with COVID-19 (Table 1). Undifferentiated connective tissue disease $(n=3)$, idiopathic uveitis $(n=3)$, Behcet's disease $(\mathrm{n}=3)$, overlap syndrome $(n=2)$, sarcoidosis $(\mathrm{n}=2)$, rheumatoid arthritis $(\mathrm{n}=2)$, systemic scleroderma $(n=1)$, Takayasu's arteritis $(\mathrm{n}=1)$, and Anti-neutrophilic cytoplasmic antibody (ANCA)-associated vasculitis $(n=1)$ were also identified as primary rheumatic diagnoses. Five patients had an additional rheumatic comorbidity: SLE+ history of macrophage activation syndrome $(\mathrm{n}=2)$, SLE+ rheumatoid arthritis $(\mathrm{n}=1)$, JIA+ Sjogren's syndrome $(\mathrm{n}=1)$, ANCA-associated vasculitis+ anti-glomerular basement membrane disease. Active disease was more commonly encountered in the hospitalized group (OR 11.83, 95\% CI [1.43-558.37]; $p=0.01$ ).

The majority of patients in both groups were taking at least one immunomodulatory medication, as detailed in Table 1. Besides those, additional immunosuppressants used in the ambulatory group included apremilast $(n=1)$, azathioprine $(n=1)$, cyclosporine $(n=1)$, tacrolimus $(n=1)$, and colchicine $(n=1)$. NSAID use was observed in $5(50 \%)$ patients in the hospitalized group and 21 (46.7\%) patients in the ambulatory group $(p=1)$. The use of medium/high-dose CS (OR 14.12; 95\% CI [2.31-106.04]; $p=0.001)$, mycophenolate (OR 8.84; 95\% CI [1.64-63.88]; $p=0.004)$ and rituximab (OR 19.40; 95\% CI [2.45-254.14]; $\mathrm{p}=0.001$ ) increased the odds of hospitalization. Patients using tumor necrosis factor-alpha inhibitors (TNFi) had decreased odds of admission (OR 0; 95\% CI $[0-1.17] ; p=0.05)$. No patients receiving intravenous immunoglobulin (IVIG) or IV pulse CS were hospitalized. No significant differences regarding use of other DMARDs were observed. Severely immunosuppressed individuals had increased odds of hospitalization (OR 34.80; 95\% CI [3.941704.26]; $p<0.001)$. 
Table 1 Demographic and baseline clinical features of children with rheumatic diseases and COVID-19

\begin{tabular}{|c|c|c|c|c|c|c|c|c|}
\hline \multirow{2}{*}{$\begin{array}{l}\text { Category } \\
\text { Demographics }\end{array}$} & \multicolumn{2}{|c|}{$\begin{array}{l}\text { Total } \\
n=55\end{array}$} & \multicolumn{2}{|c|}{$\begin{array}{l}\text { Hospitalized } \\
n=10\end{array}$} & \multicolumn{2}{|c|}{$\begin{array}{l}\text { Ambulatory } \\
n=45\end{array}$} & \multirow[t]{2}{*}{$\begin{array}{l}\text { OR } \\
(95 \% \mathrm{Cl})\end{array}$} & \multirow[t]{2}{*}{$\mathbf{P}$} \\
\hline & & & & & & & & \\
\hline Age in years & 16 & $(14-18)$ & 17 & $(16-19)$ & 16 & $(13-17)$ & & 0.07 \\
\hline Female & 43 & $(78.2)$ & 7 & $(70.0)$ & 36 & $(80.0)$ & $0.59(0.11-4.22)$ & 0.67 \\
\hline African American & 17 & $(30.9)$ & 7 & $(70.0)$ & 10 & $(22.2)$ & $7.78(1.46-55.38)$ & 0.006 \\
\hline Asian & 1 & $(1.8)$ & 0 & (0) & 1 & $(2.2)$ & $0(0-175.02)$ & 1 \\
\hline Caucasian & 31 & $(56.4)$ & 2 & $(20.0)$ & 29 & $(64.4)$ & $0.14(0.01-0.83)$ & 0.01 \\
\hline Hispanic or Latino & 6 & $(10.9)$ & 1 & $(10.0)$ & 5 & $(11.1)$ & $0.89(0.02-9.54)$ & 1 \\
\hline \multicolumn{9}{|l|}{ Baseline clinical features } \\
\hline Comorbidities & 27 & $(49.1)$ & 6 & $(60.0)$ & 21 & $(46.7)$ & $1.69(0.35-9.35)$ & 0.50 \\
\hline \multicolumn{9}{|l|}{ Primary rheumatic disease } \\
\hline Juvenile Idiopathic arthritis & 17 & $(30.9)$ & 1 & $(10.0)$ & 16 & $(35.6)$ & $0.21(0.004-1.73)$ & 0.15 \\
\hline Systemic lupus erythematosus & 14 & $(25.5)$ & 5 & $(50.0)$ & 9 & $(20.0)$ & $3.88(0.72-21.23)$ & 0.1 \\
\hline Juvenile Dermatomyositis & 6 & $(10.9)$ & 0 & (0) & 6 & $(13.3)$ & $0(0-3.94)$ & 0.58 \\
\hline Other & 18 & $(32.7)$ & 4 & $(40.0)$ & 14 & $(31.1)$ & $1.46(0.26-7.38)$ & 0.71 \\
\hline Active rheumatic disease & 28 & $(50.9)$ & 9 & $(90.0)$ & 19 & $(42.2)$ & $11.83(1.43-558.37)$ & 0.01 \\
\hline \multicolumn{9}{|l|}{ Immunomodulatory medications } \\
\hline Oral corticosteroids $<10 \mathrm{mg}$ & 8 & $(14.5)$ & 3 & $(30.0)$ & 5 & $(11.1)$ & $3.33(0.42-22.33)$ & 0.15 \\
\hline Oral corticosteroids $\geq 10 \mathrm{mg}$ & 10 & $(18.2)$ & 6 & $(60.0)$ & 4 & $(8.9)$ & $14.12(2.31-106.04)$ & 0.001 \\
\hline IV pulse corticosteroids & 3 & $(5.5)$ & 0 & $(0.0)$ & 3 & $(6.7)$ & $0(0-11.40)$ & 1 \\
\hline Hydroxychloroquine & 27 & $(49.1)$ & 7 & $(70.0)$ & 20 & $(44.4)$ & $2.89(0.56-19.35)$ & 0.18 \\
\hline Other cDMARDs ${ }^{\mathrm{a}}$ & 20 & $(36.4)$ & 2 & $(20.0)$ & 18 & $(40.0)$ & $0.38(0.03-2.22)$ & 0.3 \\
\hline Mycophenolate & 16 & $(29.1)$ & 7 & $(70.0)$ & 9 & $(20.0)$ & $8.84(1.64-63.88)$ & 0.004 \\
\hline Tofacitinib & 2 & (3.6) & 1 & $(10.0)$ & 1 & $(2.2)$ & $4.69(0.06-390.81)$ & 0.33 \\
\hline Intravenous immunoglobulin & 4 & $(7.3)$ & 0 & $(0.0)$ & 4 & $(8.9)$ & $0(0-7.15)$ & 1 \\
\hline Cyclophosphamide & 4 & (7.3) & 1 & $(10.0)$ & 3 & $(6.7)$ & $1.54(0.03-21.94)$ & 0.56 \\
\hline TNFi & 14 & $(25.5)$ & 0 & $(0.0)$ & 14 & $(31.1)$ & $0(0-1.17)$ & 0.05 \\
\hline Rituximab & 7 & $(12.7)$ & 5 & $(50.0)$ & 2 & $(2.2)$ & $19.40(2.45-254.14)$ & 0.001 \\
\hline Other bDMARDs ${ }^{\mathrm{b}}$ & 8 & $(14.5)$ & 2 & $(20.0)$ & 6 & $(13.3)$ & $0.73(0.01-7.29)$ & 1 \\
\hline None & 3 & $(5.5)$ & 0 & $(0.0)$ & 3 & $(6.7)$ & $0(0-11.40)$ & 1 \\
\hline Severe Immunosupression ${ }^{c}$ & 17 & $(32.7)$ & 9 & $(90.0)$ & 8 & $(19.0)$ & $34.80(3.94-1704.26)$ & $<0.001$ \\
\hline
\end{tabular}

Numerical variables are expressed as median (IQR), and categorical variables as $n(\%)$

Abbreviations: Cl Confidence Interval; bDMARDs biologic disease modifying anti-rheumatic drugs; CDMARDs: conventional disease modifying anti-rheumatic drugs; OR: Odds ratio; IQR: Interquartile range; TNFi: Tumor necrosis factor alpha inhibitors. ${ }^{a}$ Methotrexate $(n=12)$, leflunomide $(n=5)$, sulfasalazine $(n=3) .{ }^{b}$ Abatacept $(n=3)$, tocilizumab $(n=2)$, ustekinumab $(n=1)$, anakinra $(n=1)$, belimumab $(n=1) \cdot{ }^{c} n=52$ : Patients off immunomodulators excluded

\section{COVID-19 exposure, symptoms and diagnosis}

Exposure to a close contact with confirmed COVID-19 was reported by $5(50 \%)$ patients in the hospitalized group versus $26(57.8 \%)$ patients in the ambulatory group $(p=0.75)$.

Table 2 lists patient reported symptoms for both groups. Patients with fever, dyspnea, chest pain, rash and myalgias had increased odds of hospitalization $(p<0.05$ for each comparison).

Ten patients (18.2\%) were asymptomatic, all in the ambulatory group. Of those, one patient had a lung infiltrate in a surveillance chest tomography for metastatic
Wilms tumor, prompting SARS-CoV-2 testing. The remaining 9 patients were tested due to exposure to a close contact with confirmed COVID-19 or during routine screening at their educational institution.

Fifteen patients had confirmatory PCR testing performed at $\mathrm{CHOA}$; the rest were diagnosed at an outside facility. Of the 10 hospitalized patients, three were known to be SARS-CoV-2 positive prior to admission: patients 1 and 9 were tested one week earlier, with subsequent worsening clinical status requiring hospitalization; for patient 3, PCR test results became available while still in the emergency room. 
Table 2 Clinical presentation of COVID-19 in children with rheumatic diseases

\begin{tabular}{|c|c|c|c|c|c|c|c|c|}
\hline \multirow{2}{*}{$\begin{array}{l}\text { Symptoms } \\
\text { Fever }\end{array}$} & \multicolumn{2}{|c|}{$\begin{array}{l}\text { Total } \\
n=55\end{array}$} & \multicolumn{2}{|c|}{$\begin{array}{l}\text { Hospitalized } \\
n=10\end{array}$} & \multicolumn{2}{|c|}{ Ambulatory $n=45$} & \multirow{2}{*}{$\begin{array}{l}\text { OR } \\
\text { (95\% Cl) } \\
7.78(1.46-55.38)\end{array}$} & \multirow{2}{*}{$\begin{array}{l}\boldsymbol{P} \\
0.006\end{array}$} \\
\hline & 17 & $(30.9)$ & 7 & $(70.0)$ & 10 & $(22.2)$ & & \\
\hline Rhinorrhea/Congestion & 13 & $(23.6)$ & 2 & $(20.0)$ & 11 & $(24.4)$ & $0.78(0.07-4.79)$ & 1 \\
\hline Cough & 20 & $(36.4)$ & 4 & $(40.0)$ & 16 & (35.6) & $1.20(0.22-5.99)$ & 1 \\
\hline Dyspnea & 5 & $(9.1)$ & 4 & $(40.0)$ & 1 & $(2.2)$ & $26.28(2.17-1459.25)$ & 0.003 \\
\hline Chest pain & 6 & (6) & 4 & $(40.0)$ & 2 & $(4.4)$ & $13.20(1.53-175.79)$ & 0.007 \\
\hline Myalgias & 12 & $(21.8)$ & 5 & $(50.0)$ & 7 & (15.6) & $5.21(0.94-30.12)$ & 0.03 \\
\hline Abdominal pain & 4 & $(7.3)$ & 2 & $(20.0)$ & 2 & $(4.4)$ & $5.14(0.32-80.65)$ & 0.15 \\
\hline Anorexia/Nausea/Emesis & 8 & $(14.5)$ & 3 & $(30.0)$ & 5 & $(11.1)$ & $3.33(0.42-22.34)$ & 0.15 \\
\hline Diarrhea & 10 & $(18.2)$ & 4 & $(40.0)$ & 6 & $(13.3)$ & $4.18(0.67-24.99)$ & 0.07 \\
\hline Anosmia/Ageusia & 14 & $(25.5)$ & 2 & $(20.0)$ & 12 & (26.7) & $0.69(0.06-4.21)$ & 1 \\
\hline Rash & 5 & $(9.1)$ & 4 & $(40.0)$ & 1 & $(2.2)$ & $26.28(2.17-1459.25)$ & 0.003 \\
\hline Fatigue/Malaise & 16 & $(29.1)$ & 2 & $(20.0)$ & 14 & $(31.1)$ & $0.56(0.05-3.34)$ & 0.7 \\
\hline Sore throat & 12 & $(21.8)$ & 2 & $(20.0)$ & 10 & (22.2) & $0.88(0.08-5.50)$ & 1 \\
\hline Headache & 12 & $(21.8)$ & 2 & $(20.0)$ & 10 & $(22.2)$ & $0.88(0.08-5.50)$ & 1 \\
\hline Asymptomatic & 10 & $(18.2)$ & 0 & $(0.0)$ & 10 & (22.2) & $0(0-1.92)$ & 0.18 \\
\hline
\end{tabular}

Numerical variables are expressed as median (IQR), and categorical variables as $\mathrm{n}(\%)$

Abbreviations: Cl Confidence Interval; Odds ratio: $O R$; IQR Interquartile range

COVID-19 diagnosis was reported to Rheumatology during the acute infection period in 28 (62.2\%) ambulatory and $9(90 \%)$ hospitalized patients; the report was delayed by up 3 months for 17 (37.8\%) ambulatory patients, and by 6 weeks for $1(10 \%)$ patient admitted to an outside hospital.

\section{Complications, management and outcomes}

Immunomodulatory management during the acute COVID-19 period did not differ between groups: medications were temporarily discontinued in 5 (50\%) hospitalized patients and $16(38.1 \%)$ ambulatory patients on immunosuppression $(p=0.50)$.

Rheumatic disease flares were observed in 6 hospitalized patients versus 1 (2.2\%) ambulatory patient (OR 55.95; 95\% CI [5.16-3023.74]; $p<0.001)$. Disease flare was preceded by medication discontinuation in two patients (7 and 33). Patient 33 experienced gastrointestinal symptoms (without fever or other organ involvement) from Behcet's disease after holding adalimumab for 2 weeks; this was successfully managed by restarting the medication. Patient 7 developed flare symptoms after running out of anakinra; this improved after anakinra re-introduction and increased CS doses.

In the ambulatory group, COVID-19 treatment was largely symptomatic. Inpatient care, complications and outcomes of hospitalized patients are detailed in Table 3. Four patients required Intensive Care Unit (ICU) admission. One patient died, after having required mechanical ventilation and extracorporeal membrane oxygenation. The possibility of multisystem inflammatory syndrome in children (MIS-C) was considered but ultimately ruled out in patients 7, 9 and 10 (Table 3), based on lack of cardiac involvement, gastrointestinal symptoms explained by the presence of co-infection, and overall evolution of symptoms. No confirmed MIS-C cases were identified in this cohort. No additional late complications were encountered after a median post-COVID-19 follow-up period of 48 days.

\section{Discussion}

This study describes the presentation and outcomes of a cohort of pediatric patients with rheumatic diseases and laboratory-confirmed COVID-19. Overall, approximately $18 \%$ of our patients required hospitalization and $7.3 \%$ required ICU care. Although these rates are slightly higher than those reported in the general pediatric population, our study had a limited sample size relying mostly on voluntary reporting, and laboratory confirmation was required $[10,11]$. As such, some milder cases may have gone unreported, and untested and/or asymptomatic cases were likely excluded. Additionally, severe COVID19 has been linked African American race, which was overrepresented in the hospitalized group [2, 12]. Cardiovascular disease, a known a risk factor for hospitalization in adults, was found more frequently in the hospitalized group in our cohort, potentially having influenced these patients' outcomes [5]. Reassuringly, the vast majority of our patients did not require mechanical ventilation and survived, supporting previous reports that patients with rheumatic diseases and COVID19 overall recover well [6-9]. 


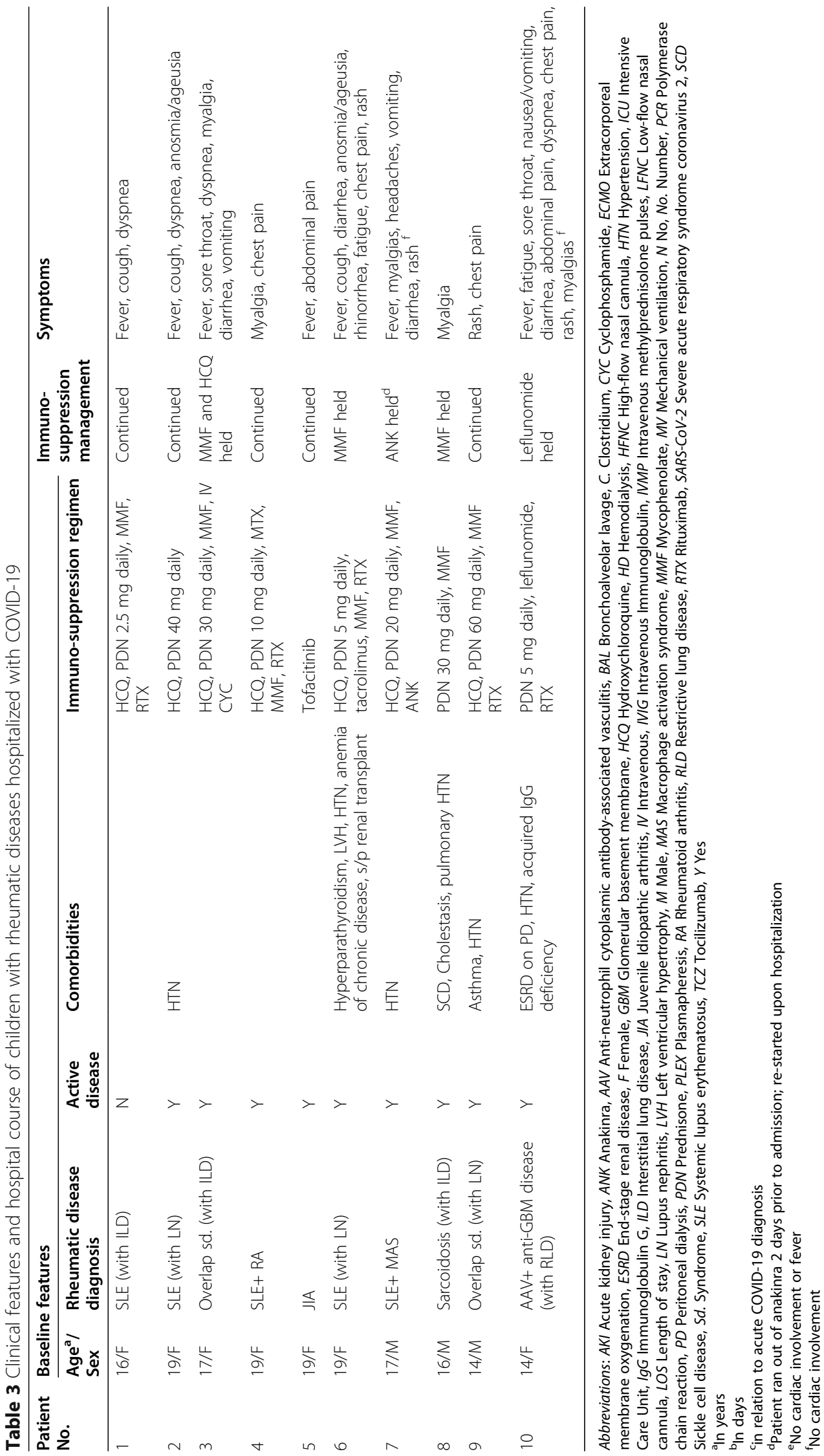




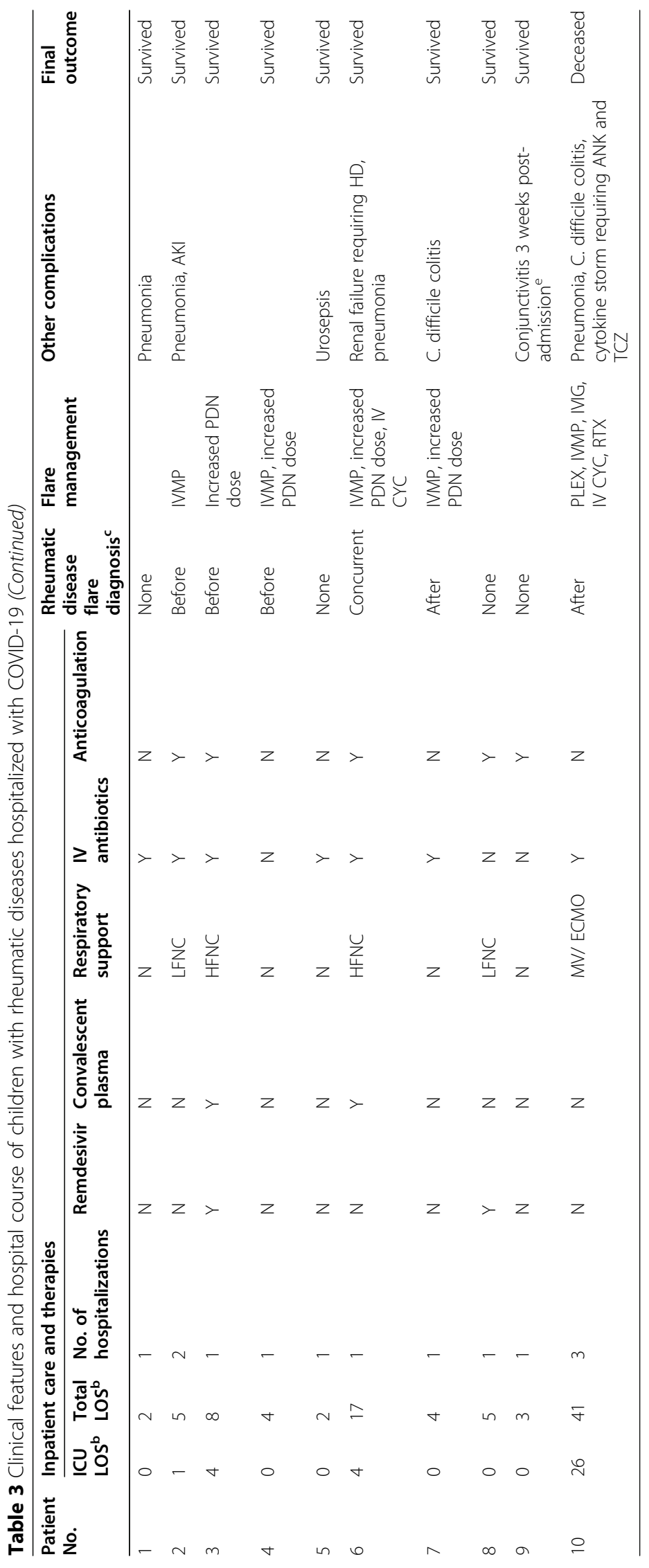


Symptoms of acute COVID-19 were similar to those described in the general pediatric population $[2,10,11]$. Symptom reporting in the hospitalized group may have been less subject to recall bias than the ambulatory group. Notably, while respiratory and gastrointestinal symptoms are typically attributable to acute infection, fever, rash and myalgias are also features of rheumatic disease flares. Fever, chest pain, myalgias, rash and dyspnea predominated in hospitalized patients; furthermore, patients with active rheumatic disease were more likely to be in the hospitalized group. These observations suggests that the interplay between rheumatic disease activity and flare, and severe COVID-19 may influence the need for admission, as proposed by Ye et al. [6]

Interestingly, while all JDM patients were managed in the ambulatory setting, SLE diagnosis was more common in the hospitalized group, although this association did not reach statistical significance. This raises the possibility of disease type playing a role in the outcomes as well. Indeed, the pattern of immune activation in patients with severe COVID-19 has been recently described, and is reported to bear many similarities to active SLE [13]. Moreover, therapy and presence of comorbidities vary by disease, offering another possible explanation for this observation.

Echoing the findings of Gianfrancesco et al., medium/ high-dose corticosteroid use was associated with increased odds of hospitalization [5]. Similar associations were observed for mycophenolate and severe immunosuppression; the latter may have been driven by the effect of medium/high-dose steroid. Patients receiving rituximab therapy were also more likely to be in the hospitalized group. While mycophenolate use has not been reported to increase the hospitalization risk in adults, rituximab use was found to be associated with higher rates of hospitalization and death from COVID-19 in a small cohort of adult patients with rheumatic diseases [14]. Although these drugs have distinct mechanisms of action, the target cells of these medications do ultimately interact to elicit the adaptive immune response; thus, it is plausible that the chronic blunting of this response could predispose the host to a more severe COVID-19 course. We were unable to elucidate if our findings were directly related to the immunosuppressive effects of these medications versus the underlying rheumatic disease and its severity, or a combination of both. Nonetheless, these results could set the ground for larger, multicenter studies to confirm these associations.

Aligning with the previously reported inverse association of TNFi and hospitalization in adults with rheumatic diseases, and the suggested absence of severe COVID-19 in a small cohort of pediatric patients with JIA on TNFi, our TNFi recipients had decreased odds of hospitalization; in fact, no patients on TNFi were hospitalized [5, 15]. Similar observations were noted for IVIG and IV pulse CS users, a previously unreported trend that merits further exploration. Therapy with other DMARDs or NSAIDs did not influence hospitalization status, comparable to adults $[5,16]$.

Importantly, management of immunomodulators did not significantly differ between groups. Withholding immunosuppression in patients with rheumatic diseases and symptomatic COVID-19 has been recommended by the American College of Rheumatology (ACR) [17]. Each case should be assessed individually, as both COVID-19 and rheumatic disease exacerbations entail risks for hospitalization, especially considering that disease flare usually warrants an increase in immunosuppression.

Our study's major strength is that, to our knowledge, this is the largest case series describing laboratoryconfirmed COVID-19 in pediatric patients with rheumatic diseases. Limitations include its retrospective nature, and the method of patient selection which precludes us from calculating population-based incidence and obtaining generalizable conclusions regarding severity compared to children without rheumatic diseases. The relatively small sample size precluded us from testing for collinearity between variables and performing multivariable analyses. Despite these limitations, we were able to achieve our aim of conveying the outcomes of COVID19 in a cohort of pediatric rheumatic disease patients, setting precedent for larger studies needed to confirm the potential associations described in this manuscript. Multicenter studies would be useful to more comprehensively define the spectrum of COVID-19 and its natural history in children with rheumatic diseases.

\section{Conclusions}

In conclusion, the need for hospitalization for COVID19 in this cohort of pediatric patients with rheumatic diseases was associated with African American race, cardiovascular disease, active rheumatic disease, medium/ high-dose prednisone use, mycophenolate use, rituximab use and severe immunosuppression. Fever, dyspnea, chest pain, rash and myalgias were high-risk symptoms for hospitalization which should be closely monitored when present. Decisions to continue or discontinue rheumatic disease therapy should be made on a case-bycase basis, following current ACR guidelines.

\footnotetext{
Abbreviations

ACR: American College of Rheumatology; CHOA: Children's Healthcare of Atlanta; Cl: Confidence Interval; COVID-19: Coronavirus Disease 19; CS: Corticosteroids; DMARDs: Disease modifying anti-rheumatic drugs; IRB: Institutional Review Board; ICU: Intensive Care Unit; IQR: Interquartile range; IV: Intravenous; IVIG: Intravenous Immunoglobulin; JDM: Juvenile Dermatomyositis; JIA: Juvenile Idiopathic Arthritis; JADAS: Juvenile Arthritis Disease Activity Score; MIS-C: Multisystem inflammatory syndrome in children; NSAIDs: Non-Steroidal anti-inflammatories; OR: Odds ratio; PCR: Polymerase chain reaction; SARS-CoV-2: Severe Acute Respiratory Syndrome Coronavirus 2; SLE : Systemic lupus erythematosus; SLEDAI-2
} 
K: Systemic Lupus Erythematosus Disease Activity Index-2000; TNFi: Tumor necrosis factor alpha inhibitors

\section{Acknowledgements}

We would like to thank Drs. Amit Thakral, Cynthia Manos, Elaine Flanagan, Angela Taneja, Baruch Goldberg, Omkar Phadke, Meghan Nelson and Sabina Kennedy for their contribution in the identification of cases.

\section{Authors' contributions}

DSVN and SP contributed to study design, data collection, data analysis; drafted and revised the manuscript critically for intellectual content and gave the final approval for the published version. KRS contributed to data collection and data analysis, revised the manuscript critically for intellectual content and gave the final approval for the published version. CAR, SC, and AK contributed to the study design and data analysis, revised the manuscript critically for intellectual content and gave the final approval for the published version.

\section{Funding}

Dr. Prahalad is supported in part, by the Marcus Foundation Inc., Atlanta. No additional funding was secured for this study.

\section{Availability of data and materials}

The datasets used and/or analyzed during the current study are available from the corresponding author on reasonable request.

\section{Declarations}

\section{Ethics approval and consent to participate}

The present study was approved by the Institutional Review Board (IRB) at CHOA via waiver of informed consent (IRB number STUDY00000771).

\section{Consent for publication}

Not applicable.

\section{Competing interests}

Dr. Rostad reports grants from BioFire, grants from Janssen, grants from Moderna, grants from Micron, grants from Medlmmune, grants from PaxVax, outside the submitted work; In addition, Dr. Rostad has a patent US20180333477A1 with royalties paid to Emory University.

Dr. Prahalad reports serving on a Macrophage Activation Syndrome Adjudication Committee for Novartis Pharmaceuticals. In addition, he was a consultant to Sigilon Therapeutics on immune mediated diseases. These have no bearing on the work reported herein.

The remaining authors have no conflicts of interest to declare.

\section{Author details}

'Department of Pediatrics, Division of Pediatric Rheumatology, Emory University School of Medicine, Atlanta, USA. ${ }^{2}$ Children's Healthcare of Atlanta, Atlanta, USA. ${ }^{3}$ Department of Pediatrics, Division of Pediatric Infectious Diseases, Emory University School of Medicine, Atlanta, USA. ${ }^{4}$ Department of Medicine, Division of Rheumatology, Emory University School of Medicine, Atlanta, USA. ${ }^{5}$ Department of Pediatrics, Division of Hematology, Oncology and BMT, Emory University School of Medicine, Atlanta, USA. ${ }^{6}$ Department of Human Genetics, Emory University School of Medicine, Atlanta, USA.

\section{Received: 10 January 2021 Accepted: 18 May 2021}

\section{Published online: 21 June 2021}

\section{References}

1. Shen Q, Guo W, Guo T, Li J, He W, Ni S, et al. Novel coronavirus infection in children outside of Wuhan. China Pediatr Pulmonol. 2020;55(6):1424-9. https://doi.org/10.1002/ppul.24762.

2. DeBiasi RL, Song X, Delaney M, Bell M, Smith K, Pershad J, et al. Severe coronavirus Disease-2019 in children and young adults in the Washington DC, Metropolitan Region. J Pediatr. 2020;223:199-203 e1.

3. Danza A, Ruiz-Irastorza G. Infection risk in systemic lupus erythematosus patients: susceptibility factors and preventive strategies. Lupus. 2013;32: 1286-94.

4. Beukelman T, Xie F, Chen L, Baddley JW, Delzell E, Grijalva CG, et al. Rates of hospitalized bacterial infection associated with juvenile idiopathic arthritis and its treatment. Arthritis Rheum. 2012;64(8):2773-80. https://doi.org/10.1 002/art.34458.

5. Gianfrancesco M, Hyrich KL, Al-Adely S, Carmona L, Danila MI, Gossec L, et al. Characteristics associated with hospitalisation for COVID-19 in people with rheumatic disease: data from the COVID-19 global rheumatology Alliance physician-reported registry. Ann Rheum Dis. 2020;79(7):859-66. https://doi.org/10.1136/annrheumdis-2020-217871.

6. Ye C, Cai S, Shen G, Guan H, Zhou L, Hu Y, et al. Clinical features of rheumatic patients infected with COVID-19 in Wuhan. China Ann Rheum Dis. 2020;79(8):1007-13. https://doi.org/10.1136/annrheumdis-2020-217627.

7. Michelena X, Borrell H, Lopez-Corbeto M, Lopez-Lasanta M, Moreno E, Pascual-Pastor M, et al. Incidence of COVID-19 in a cohort of adult and paediatric patients with rheumatic diseases treated with targeted biologic and synthetic disease-modifying anti-rheumatic drugs. Semin Arthritis Rheum. 2020;50(4):564-70. https://doi.org/10.1016/j.semarthrit.2020.05.001.

8. Koker O, Demirkan FG, Kayaalp G, Cakmak F, Tanatar A, Karadag SG, et al. Does immunosuppressive treatment entail an additional risk for children with rheumatic diseases? A survey-based study in the era of COVID-19. Rheumatol Int. 2020;40(10):1613-23. https://doi.org/10.1007/s00296-020-04 663-9.

9. Filocamo G, Minoia F, Carbogno S, Costi S, Romano M, Cimaz R. Absence of severe complications from SARS-CoV-2 infection in children with rheumatic diseases treated with biologic drugs. J Rheumatol. 2020:jrheum.200483. https://doi.org/10.3899/jrheum.200483.

10. US Department of Health and Human Services/Centers for Disease Control and Prevention: CDC COVID-19 Response Team: Coronavirus Disease 2019 in Children - United States, February 12-April 2, 2020. Morbidity and Mortality Weekly Report. 2020;69(14):422-6.

11. Lu X, Zhang L, Du H, Zhang J, Li Y, Qu J, et al. SARS-CoV-2 infection in children. N Engl J Med. 2020;382(17):1663-5. https://doi.org/10.1056/NEJMc2 005073.

12. Gianfrancesco MA, Leykina LA, Izadi Z, Taylor T, Sparks JA, Harrison C, et al. Race/ethnicity association with COVID-19 outcomes in rheumatic disease: data from the COVID-19 global rheumatology Alliance physician registry. Arthritis Rheumatol. 2020;73:374-80

13. Woodruff MC, Ramonell RP, Nguyen DC, Cashman KS, Saini AS, Haddad NS, et al. Extrafollicular B cell responses correlate with neutralizing antibodies and morbidity in COVID-19. Nat Immunol. 2020;21(12):1506-16. https://doi. org/10.1038/s41590-020-00814-z.

14. Loarce-Martos J, Garcia-Fernandez A, Lopez-Gutierrez F, Garcia-Garcia V, Calvo-Sanz L, Del Bosque-Granero I, et al. High rates of severe disease and death due to SARS-CoV-2 infection in rheumatic disease patients treated with rituximab: a descriptive study. Rheumatol Int. 2020;40(12):2015-21. https://doi.org/10.1007/s00296-020-04699-x.

15. Marino A, Romano M, Gattinara M, Cimaz R. Patients with juvenile idiopathic arthritis on TNF inhibitors exposed to COVID-19 family members. Semin Arthritis Rheum. 2020;50(6):1214-5. https://doi.org/10.1016/j.semarthrit.2020. 09.012.

16. Scirè CA, Carrara G, Zanetti A, Landolfi G, Chighizola C, Alunno A, et al. COVID-19 in rheumatic diseases in Italy: first results from the Italian registry of the Italian Society for Rheumatology (CONTROL-19). Clin Exp Rheumatol. 2020;38(4):748-53.

17. Wahezi DM, Lo MS, Rubinstein TB, Ringold S, Ardoin SP, Downes KJ, et al. American College of Rheumatology Guidance for the Management of Pediatric Rheumatic Disease during the COVID-19 pandemic: version 1. Arthritis Rheum. 2020:1-11

\section{Publisher's Note}

Springer Nature remains neutral with regard to jurisdictional claims in published maps and institutional affiliations. 